\title{
Self-Resonant Microlasers of Colloidal Quantum Wells Constructed by Direct Deep Patterning
}

\author{
Negar Gheshlaghi, Sina Foroutan-Barenji, Onur Erdem, Yemliha Altintas, Farzan Shabani, \\ Muhammad Hamza Humayun, and Hilmi Volkan Demir*
}

Cite This: Nano Lett. 2021, 21, 4598-4605

Read Online

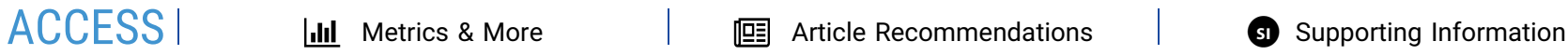

ABSTRACT: Here, the first account of self-resonant fully colloidal $\mu$-lasers made from colloidal quantum well (CQW) solution is reported. A deep patterning technique is developed to fabricate well-defined high aspect-ratio on-chip CQW resonators made of grating waveguides and in-plane reflectors. The fabricated waveguide-coupled laser, enabling tight optical confinement, assures in-plane lasing. CQWs of the patterned layers are closedpacked with sharp edges and residual-free lifted-off surfaces. Additionally, the method is successfully applied to various nanoparticles including colloidal quantum dots and metal nanoparticles. It is observed that the patterning process does not affect the nanocrystals (NCs) immobilized in the attained patterns and the different physical and chemical properties of the NCs remain

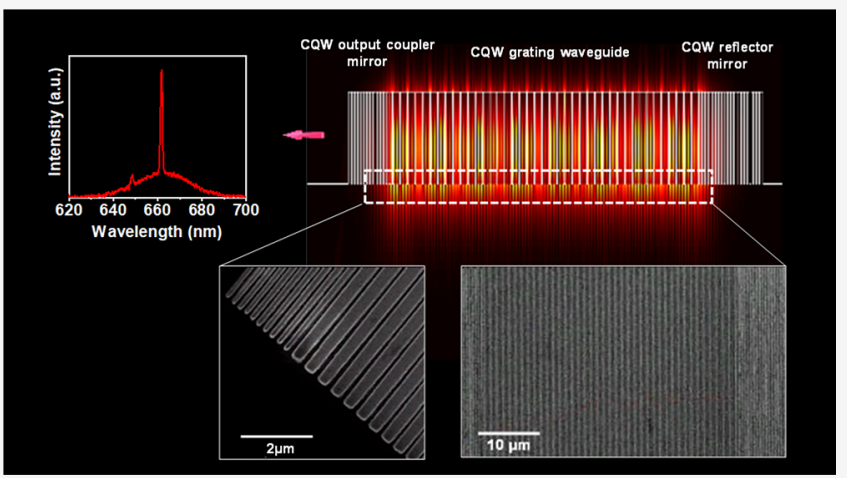
pristine. Thanks to the deep patterning capability of the proposed method, patterns of NCs with subwavelength lateral feature sizes and micron-scale heights can possibly be fabricated in high aspect ratios.

KEYWORDS: semiconductor nanocrystals, direct nanopatterning, UV-induced ligand exchange, electron beam lithography, microlaser, optical nanocircuit, colloidal quantum wells

\section{INTRODUCTION}

Quantum photonics holds great promise for future technologies such as secure communication, quantum computation, quantum simulation, and quantum metrology. The technologies of semiconductor integrated circuits and electronic devices are rapidly approaching their fundamental limits in terms of both processing speed and data transmission rate. One way to overcome this limitation is to employ photons rather than electrons in the functional processing components. ${ }^{1,2}$ Although tremendous progress has been made toward the development of scalable miniature circuits, integration of a versatile optical source that couples light into the waveguides as one of the main elements of a photonic circuit is highly desirable because it would avoid the limitations of otherwise delivering light from a bulky external source to the nanometer scale. Microlasers $(\mu$ lasers) may provide a viable option, as they perform singlemode photon emission and allow for the generation of coherent radiation within an extremely small footprint. However, despite many demonstrations of this class of lasers based on III-V materials and silicon, the required high growth temperatures and large lattice mismatch between laser material and substrate in monolithic integration as well as the difficulties in precise surface flatness required for bonding fabricated lasers on a substrate, and the realization of low cryogenic operation temperature of these lasers, present a formidable array of challenges to current fabrication technologies. ${ }^{3}$

Colloidal semiconductor nanocrystals (NCs) are emerging as new optoelectronic materials that have recently been commanding considerable attention in photonics thanks to their size-tunable bandgaps, strong light absorption, narrow spectral emission, chemical stability, and easy processability. ${ }^{4-7}$ Compared to the epitaxial growth of III-V quantum wells, solution-processing of NCs offers low-cost manufacturability in optoelectronics. A variety of types of colloidal quantum dots (QDs) and their two-dimensional counterparts, colloidal quantum wells (CQWs), have been widely explored as optical gain medium and several successful optical feedback configurations including Fabry-Pérot cavity, whispering gallery mode (WGM) and distributed feedback (DFB) ${ }^{8-18}$ lasers have been developed recently. However, these reports mostly focused on the performance of individual devices, and despite the confirmation of NCs as optical-gain media in these

Received: February 2, 2021

Revised: May 10, 2021

Published: May 24, 2021 
a

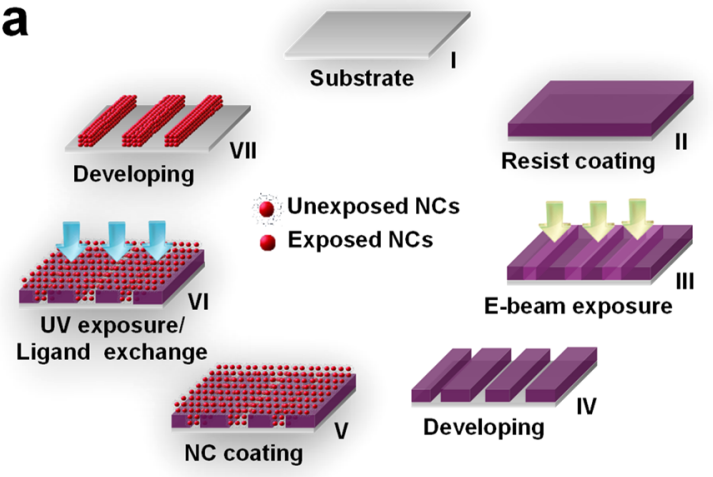

C

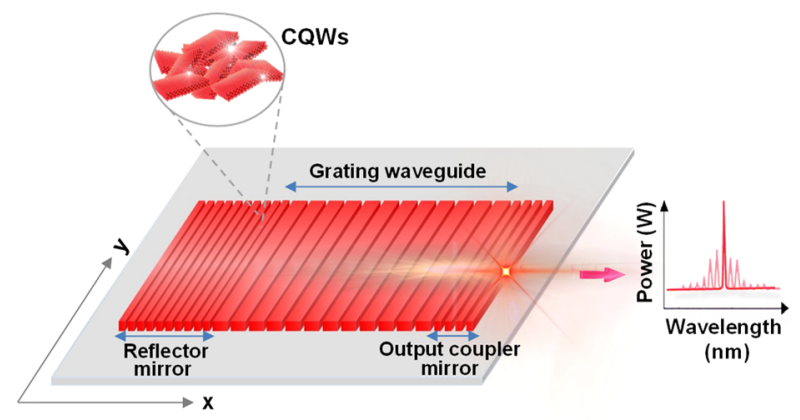

b
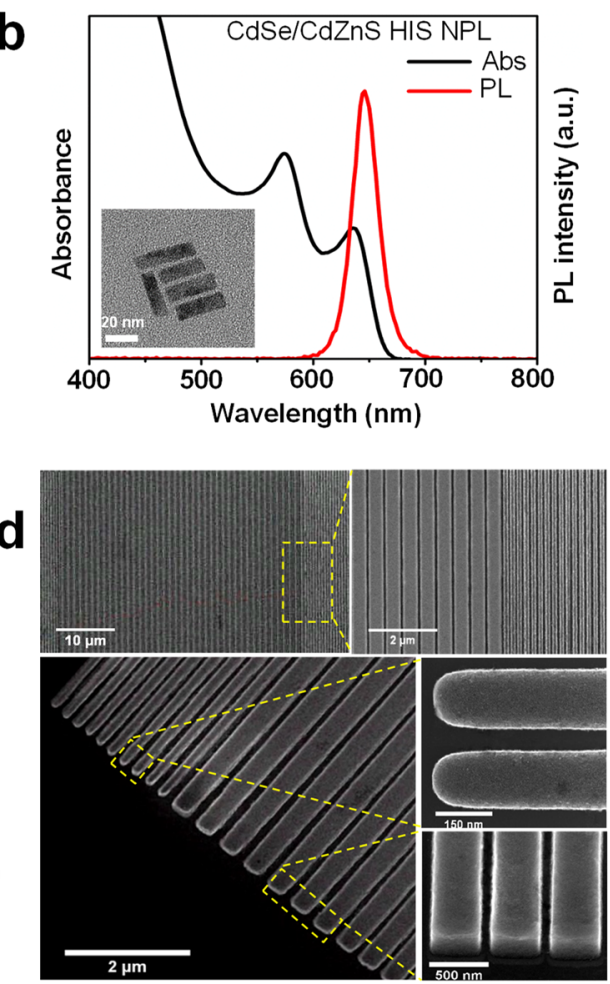

Figure 1. (a) Flow of the processing steps for fabricating patterned NC films. (b) PL and absorption spectra of CdSe/Cd0.25Zn0.75S core/HIS CQWs. The inset shows high-resolution transmission electron microscopy (HR-TEM) image of the CQWs (the scale bar is $20 \mathrm{~nm}$ ). (c) Schematic view of the designed self-resonant all-colloidal laser made of DBR waveguides and mirrors. (d) Scanning electron micrograph of the final colloidal device structure at different magnifications. (Mirrors and emitters have 200 and $500 \mathrm{~nm}$ pitches, respectively).

structures, a practical and fundamental way to develop a fully integrated on-chip waveguide-coupled $\mu$-laser sources has yet to be achieved. A challenge in the NC solution-based device is to create high-density, close-packed, well-defined colloidal nanopatterns with controllable film thickness. Different methods of NC film patterning have been considered and a few successful NC patterning techniques including UV/ photocuring patterning, ${ }^{19-22}$ microcontact printing, ${ }^{23,24}$ and patterning through electrospinning ${ }^{25}$ have been studied. Still, in most of these methods, colloidally synthesized NCs capped with long-chain organic ligands are patterned directly or blended with organic polymers. Unfortunately, these organic ligands and additives adversely affect device performance. These act as insulating barriers that impede charge or heat transport between NCs and decrease film density, because they do not form close-packed films, leading to limited pattern thickness and resolution. These issues become more critical when NCs are used as gain material. The gain value is dependent on the packing density of the NCs inside the surrounding matrix. Low packing density reduces the gain coefficient as well as refractive index of these layers and then their optical mode confinement. ${ }^{26}$

Here, we demonstrate the first account of on-chip selfresonant all-NC $\mu$-laser that incorporates patterned NCs as a gain medium between ultracompact patterned NCs as mirrors. The proposed structure has the potential to be coupled to a waveguide to be used in chip-scale photonic devices. Unlike exploitation of simple fabrication methods ${ }^{27,28}$ such as "coffeestain" cavities, ${ }^{29-31}$ which have resulted in limited control over the cavity geometry, our proposed fabrication approach enables tight confinement of light, significantly enhances the directionality of the output beam, and assures in-plane laser emission propagation. Here, the devised fully colloidal laser architecture relies on self-resonating distributed Bragg reflector (DBR) grating waveguide as the gain section integrated in plane with a pair of reflectors, one of which is a highly reflective (100\%) DBR grating, whereas the other is defined as a moderately reflective (90\%) DBR outcoupler. All these parts are directly patterned using the same NCs, a specific type of CQWs synthesized in core/hot-injection shell (HIS) both as gain and dielectric media. Indeed, the fabrication of such highquality resonators requires close-packed, high-contrast, and contamination-free subwavelength patterning technique.

In this study, we developed a novel deep-patterning method to process NC films in close packing. This technique combines electron beam lithography with UV-induced ligand exchange and enables fabrication of the nanopatterned NC structures. The original long bulky insulating ligands are replaced with shorter compact ones to obtain close-packed patterned films, while intrinsic physical and chemical properties of the NCs are not altered. Using the proposed direct deep-patterning technique, high-aspect-ratio NC patterns are fabricated. Lateral feature sizes below $100 \mathrm{~nm}$ resolution, limited by the available electron beam lithography (EBL) apparatus are achieved, while feature thicknesses up to $1.5 \mu \mathrm{m}$ were reproducibly obtained. These achievable dimensions conveniently allow for construction of fully colloidal self-resonant $\mu$-laser with grating waveguides as gain medium as well as a pair of in-plane mirrors made of DBRs.

From the fabricated $\mu$-laser, the low lasing threshold was achieved under femtosecond optical pumping. The lasing action was characterized via measuring the photoluminescence 

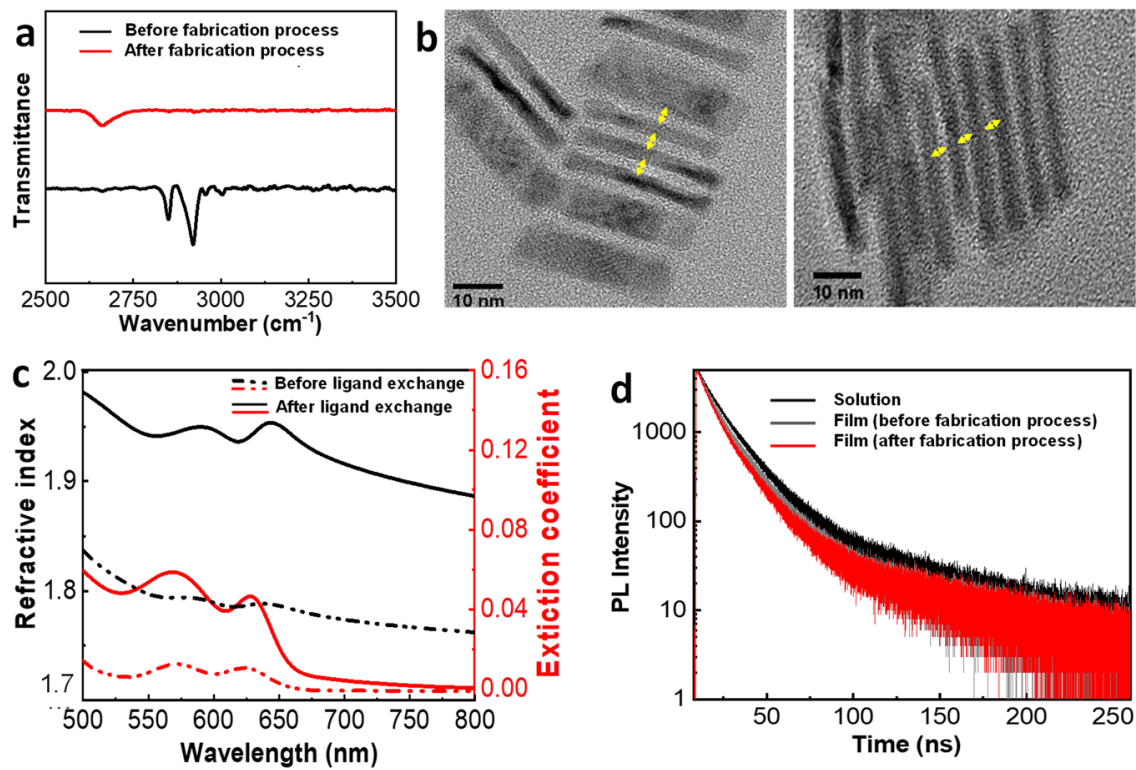

Figure 2. (a) FTIR spectra of CQWs capped with native oleate ligands and after UV exposure on Si substrate. (b) TEM images of CQWs before and after UV exposure. (c) Real and imaginary effective refractive indices of the CQW thin film before and after ligand exchange, measured via spectroscopic ellipsometry. (d) PL decay kinetics of CQW ensembles before and after the patterning process.

(PL) intensity versus pump power. The achieved lasing threshold is comparable to those obtained using external DFB and WGM schemes with NCs. ${ }^{14,32-36}$ In the case of using such an on-chip self-resonant $\mu$-laser cavity, the high refractive index contrast between the patterned CQWs and the air increases the optical mode confinement in the gain medium, which makes it relatively easier to lase. Low-threshold lasing is indicative of efficient in-plane feedback and low-loss waveguiding, and therefore a further confirmation of the high structural fidelity of our deep-patterned films.

\section{RESULTS AND DISCUSSION}

The fabrication of self-resonator $\mu$-laser started with the patterning of $\mathrm{CdSe} / \mathrm{Cd}_{0.25} \mathrm{Zn}_{0.75} \mathrm{~S}$ core/hot-injection shell (HIS) CQWs, which is synthesized using modifications according to the synthesis recipe reported in our group's recent studies ${ }^{37,38}$ (see the Supporting Information). To assemble the CQWs into nanoscale patterns, we used EBL to pattern resist followed by deposition of functionalized photosensitive NCs and UV exposure for ligand replacement and lift-off. Figure 1a shows a schematic of the process flow. Figure $1 \mathrm{~b}$ depicts photoluminescence (PL) spectrum of these CQWs along with their absorption spectrum at the emission peak centered at $647 \mathrm{~nm}$ with a fwhm of $25 \mathrm{~nm}$.

As a suitable candidate to conduct measurements in visible light range, a well-cleaned fused silica substrate was used on which the $\mu$-laser was fabricated. ${ }^{39}$ After spin-coating of an electron beam resist on the prepared substrate, EBL was used to create the designed $\mu$-laser structure (Figure 1a, steps I, II, III, and IV). A detailed description of the fabrication steps can be found in SI. A scheme of the $\mu$-laser consisting of emitter and mirror is shown in Figure 1c.

Complete coverage of a patterned trench structure with colloidal NCs is the next crucial step in such a way that voids and vacant sites between NCs were avoided. If not, the lasing performance of the fabricated structure would be drastically affected. Indeed, when colloidal NCs are assembled into NC solids, the long, bulky, insulating ligands prevent close packing of these NCs, and consequently, undesired weak interparticle coupling adversely affects device performance. One of the promising solutions to increase the packing density and coupling strength of the NCs is to exchange the native capping ligand on the NC with smaller molecules either in solution and before film formation or by immersing their dried film in a solution containing smaller molecules. ${ }^{40,41}$ As an alternative to these approaches, UV light was used to initiate ligand replacement. There have been several reports that have endorsed and demonstrated the capability of this technique. Jun et al. previously demonstrated the possibility of crosslinking the unsaturated double bonds between oleic acid (OA) ligands under UV illumination. ${ }^{42}$ David et al. showed the decomposition of $\mathrm{CS}_{2} \mathrm{~N}_{3}$ ligands to thiocyanate under UV exposure. ${ }^{43} \mathrm{Kim}$ et al. reported an approach to functionalize $\mathrm{NCs}$ by incorporation of the functional t-butoxycarbonyl ( $t$ BOC) ligand, which has an acid-labile moiety and activates upon UV exposure. ${ }^{22}$ Recently, Talapin and collaborators have reported a comprehensive study on series of photochemically active surface ligands for NCs. They designed a broad class of photochemically active ligands using various photon energies including DUV, near-UV, blue, and visible light where the change in surface chemistry was successfully exploited for direct patterning of NCs. ${ }^{20}$ Although the direct lithography of NCs requires fewer steps with respect to conventional lithography, the reported patterns suffered from imprecise control of film thickness and the remaining residual layer on the surface. However, in the NC solution-based device fabrication process, fabrication of contamination-free, closedpacked, well-defined nanopatterns with controllable film thickness is essential, which remains a challenge to date.

Here, we have circumvented the aforementioned problems with combining electron beam lithography and UV-induced ligand exchange procedure. In the film formation step (Figure la, steps V and VI), the well-cleaned NCs capped with organic ligands such as $\mathrm{OA}$ and oleylamine were dispersed in octane and functionalized by incorporation of (C6H5) ${ }_{3} \mathrm{~S}+\mathrm{OTf}^{-}$, (triphenyl sulfonium triflate) photo acid generating (PAG) 

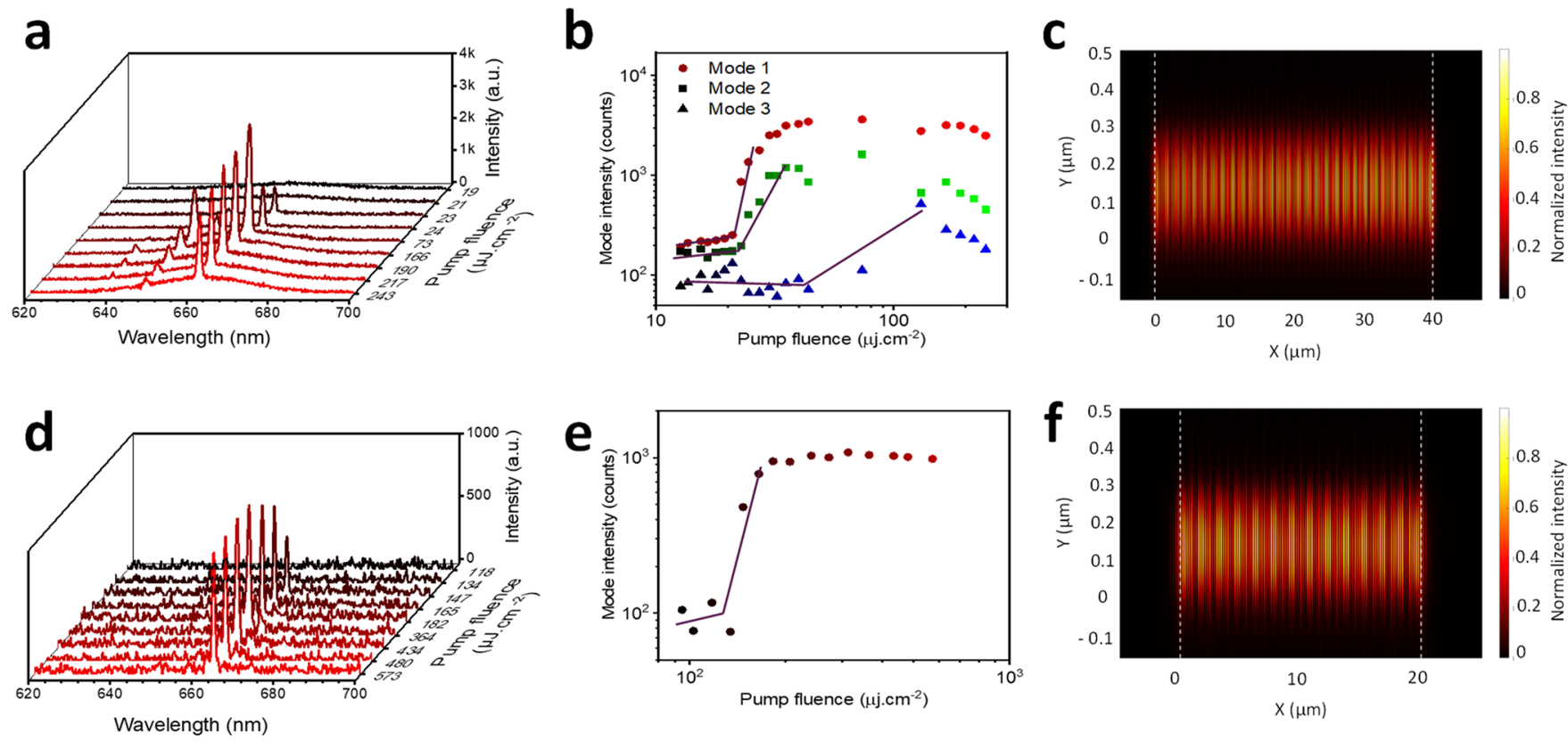

Figure 3. (a) PL spectra of the deep-patterned CQWs under various excitation intensities. Three lasing modes at 662, 650, and 638 nm appear with onsets of $\sim 21, \sim 23$, and $\sim 70 \mu \mathrm{J} / \mathrm{cm}^{2}$, respectively. (b) Peak intensities of the three lasing modes at $\sim 662$ (red circles), $\sim 650$ (green squares), and $\sim 638 \mathrm{~nm}$ (blue triangles). (c) Simulated cavity mode intensity profile for the dominant mode at $664 \mathrm{~nm}$. Dashed lines specify the $40 \mu \mathrm{m}$ long active emitter region. Aside from the leaks into the substrate and air, the mode is confined to the cavity formed by the mirrors. As expected, the lasing frequency is pulled toward the PL peak. (d) PL spectra of the deep-patterned CQWs under pulsed excitation of various intensities. (e) Peak intensity of the spectra as a function of the pump fluence. The steep increase in the curve corresponds to the onset of the lasing at a threshold of $\sim 140 \mu \mathrm{J} / \mathrm{cm}^{2}$, followed by the saturation of the peak intensity. (f) Simulated cavity mode intensity profiles for the dominant mode at $\sim 663 \mathrm{~nm}$. Dashed lines specify $20 \mu \mathrm{m}$ long active region. Like in c, the energy is mostly confined to the cavity, with small leaks in the vertical direction.

surface ligands. According to established procedures, PAG molecules were dissolved in $\mathrm{EtOH}$ and mixed with the $\mathrm{NC}$ solution (5\%, by weight). ${ }^{19}$ The photochemically active solution is deposited on predefined patterns resist and let to dry. Subsequently, Under UV exposure, (C6H5) ${ }_{3} \mathrm{~S}+\mathrm{OTf}^{-}$ molecules decompose and generate a strong acid, triflic acid (HOTf). The strong acid interacts with the NCs surface and the photogenerated protons efficiently attack lyophilic ligands and replace them with $\mathrm{OTf}^{-}$groups. As a very weak nucleophile, triflate forms an ion pair rather than a covalent bond with metal sites at the NC surface. The new OTf ligands represent a substantial decrease in ligand size. The liftoff process was performed by immersion of the sample in acetone/octane mixture under mild ultrasonic stirring (Figure 1a, step VII). The product of the mentioned steps is a robust, consistently crack-/void-free film with strong adhesion to the surface. Atomic force micrograph (AFM) shows a uniform height of the deep-patterned CQW films with no chip-off after development of the pattern (Figure S1). High-resolution scanning electron microscopy of fabricated CQW self-resonant distributed Bragg reflector grating structure at different magnifications is presented in Figure 1d. Unlike regular methods, UV-assisted ligand exchange offers a substantial simplification of the processing steps.

Fourier transform infrared (FTIR) spectroscopy was used to monitor OA left on the deep-patterned film. The exchange can be followed by comparing the vibrational spectrum before and after the exchange. Figure $2 \mathrm{a}$ shows diagnostic signals for native organic ligands at $2800-3000 \mathrm{~cm}^{-1}$ for $\mathrm{C}-\mathrm{H}$ stretching mode, ${ }^{44}$ which CQWs-OA thin films exhibited before UV exposure, were drastically suppressed after UV exposure. The new shorter ligands form an ion pair rather than a covalent bond with metal sites at the NC surface and lead to a substantial decrease in the interparticle separation. The comparison of TEM images in Figure $2 b$ shows the synthesized CQWs with lyophilic ligands and large interparticle separation $(2.15 \mathrm{~nm})$ retained their size and morphology after UVinduced ligand exchange and the gaps between individual CQWs are reduced to $1.08 \mathrm{~nm}$. Switching to shorter ligands has resulted in improved performance of solution-processed solar cells, ${ }^{45}$ photodetectors, ${ }^{46}$ and field-effect transistors (FETs). ${ }^{47}$ Similarly, the refractive index of the deep-patterned NC films plays a pivotal role in photonic applications. To determine the effective complex refractive index of the CQW films before and after UV-induced ligand exchange, variableangle spectroscopic ellipsometry was used. Unlike the intrinsic refractive index, the effective refractive index is sensitive to morphology, as it accounts for the NCs, ligands, and void space, which make up the overall film. The results in Figure $2 \mathrm{c}$ show that the films subjected to UV-induced ligand exchange procedure have a higher effective complex refractive index because the decreased bound ligand chain length during pattering process led to the increment of inorganic volume fraction, optical density, and associated packing fraction of these films. The effective complex refractive index of NC solid films is dominated by the fill fraction of NCs, with only secondary influence from interparticle interaction and vary because of the particle size, the ligand chain length, and the deposition process. $^{48,49}$

Figure $2 \mathrm{~d}$ shows the recorded PL decay curves for both of the samples that were used to estimate the effect on the PLQE following the pattern formation and subsequent ligand exchange. The lifetime of the thin-film sample drops was comparable to that of the solution sample. However, there is a 
a

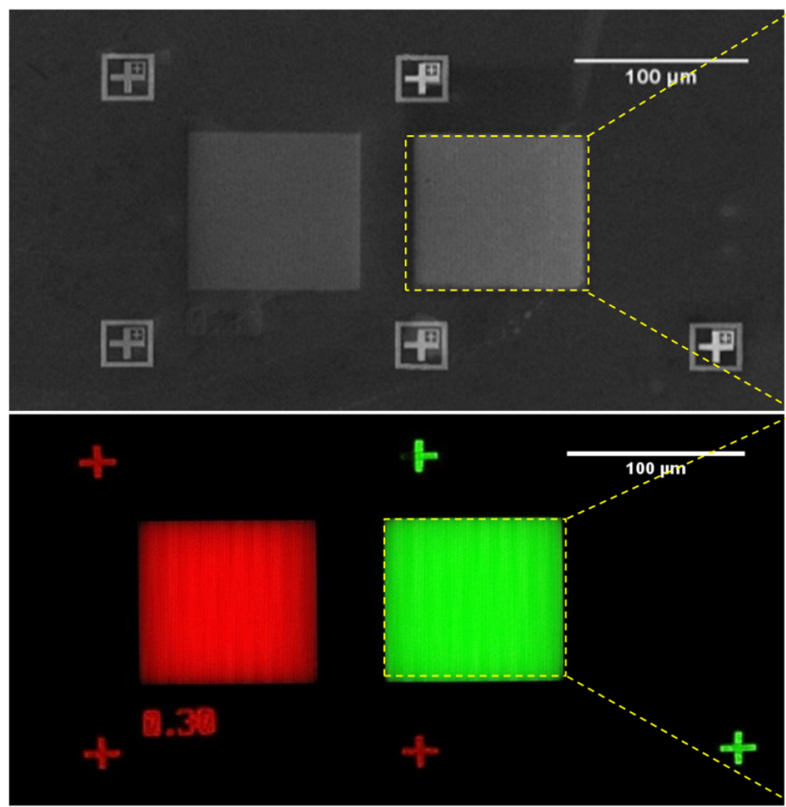

b

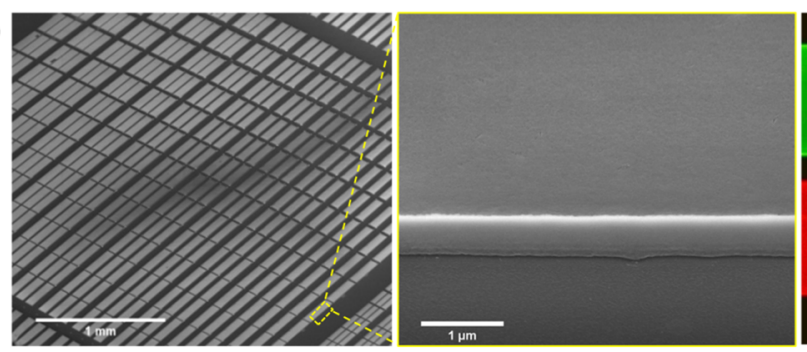

C
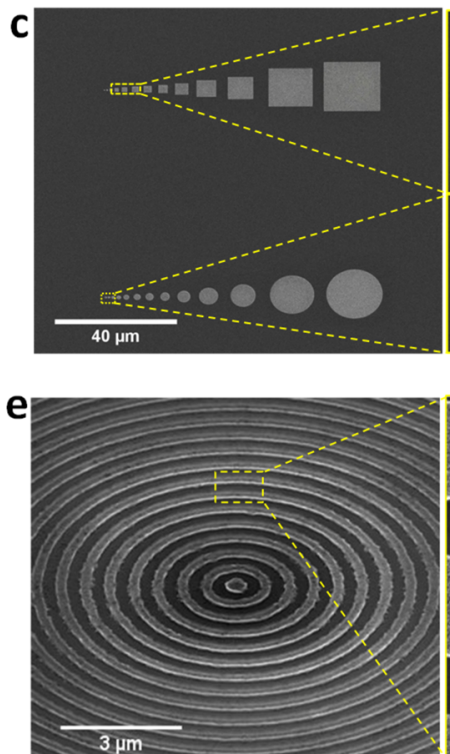
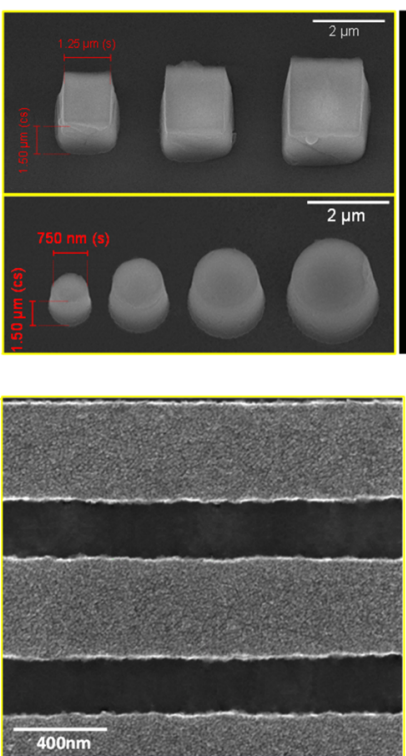
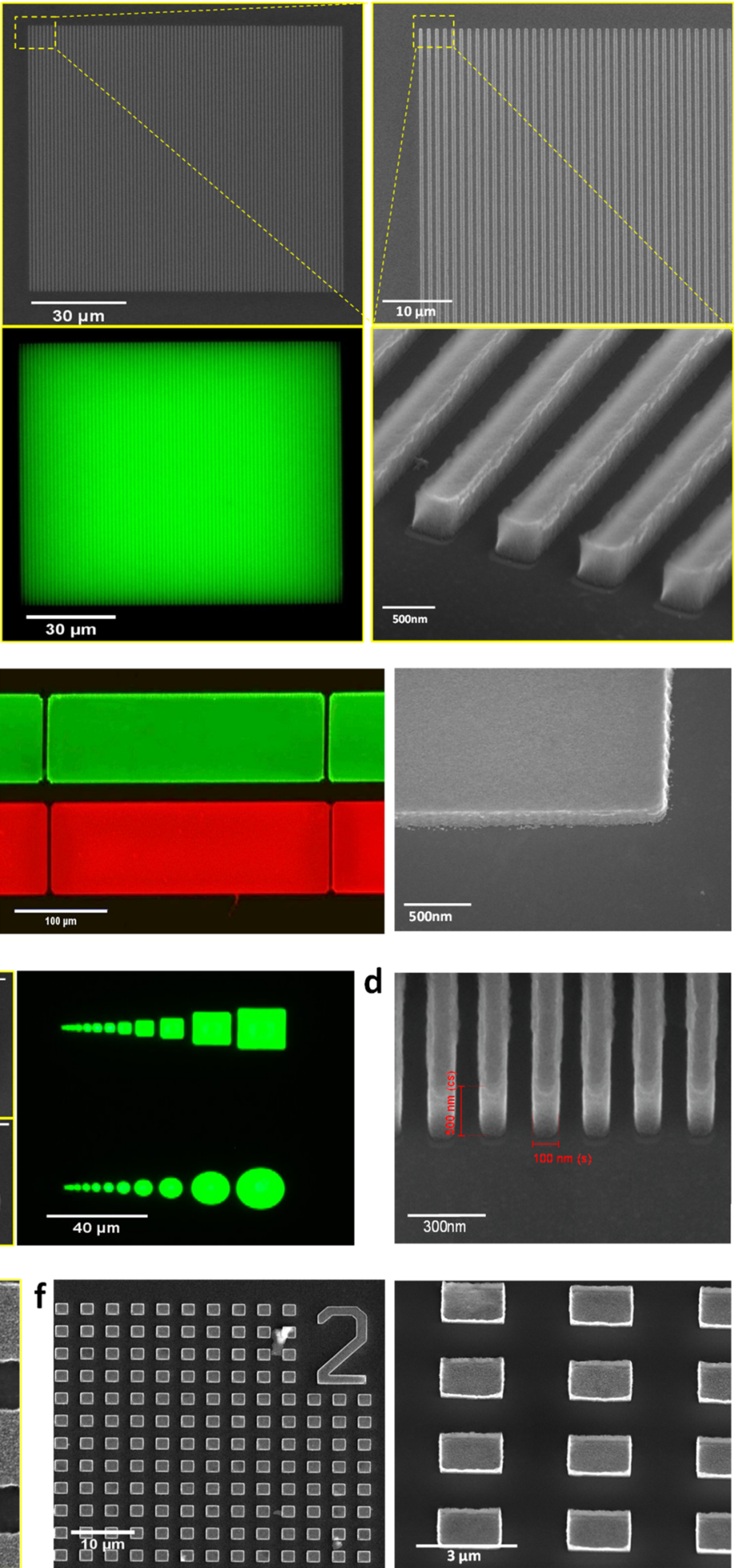

Figure 4. Bilayer of red-emitting CdSe/CdZnS CQWs and green-emitting CdSe/ZnS QDs patterned with (a) EBL, (b) photolithography, and imaged with SEM and PL microscopy. (c) High-quality and thick green-emitting CdSe/ZnS QDs patterned with EBL and imaged with SEM and PL microscopy. (d) High-quality and thick red-emitting CdSe/CdZnS CQWs patterned by EBL. (e) Bragg gratings patterns of red-emitting CdSe/ CdZnS CQWs and (f) Au NPs patterned by EBL and imaged with SEM.

marginal difference before and after the patterning process of the thin film, albeit a slight decrement in the lifetime, suggesting insignificant modification of the PLQE (see Table S1). To check potential damage on the CQWs during the fabrication process, we compared QE of the CQW films before and after the fabrication process and found the deviation is in the range of a few percent (see Figure S3).

The design parameters of mirror and emitter slab thicknesses and lateral dimensions, waveguide width, and emission wavelength of the $\mu$-laser structure were carefully chosen to achieve high optical mode confinement and enable a 
low lasing threshold via full electromagnetic solution using finite difference time-domain (FDTD) simulations. The resulting self-resonant CQW $\mu$-laser attained by our direct deep-patterning approach forms an in-plane version of a Fabry-Pérot resonator consisting of DBR-based self-resonating gain section laterally sandwiched between a pair of DBR reflective regions. The gain part is composed of an array of deep-patterned CQW gratings with a film thickness of $300 \mathrm{~nm}$. The mirrors are made of the same NCs, designed with a 200 $\mathrm{nm}$ pitch from multiple layers of alternating CQWs and air resulting in periodic variation in the refractive index and act as a high-quality reflector. The emitters are designed with a 500 $\mathrm{nm}$ pitch to effectively confine and guide the emission of the CQWs at $650 \mathrm{~nm}$. To achieve a higher level of optical confinement, better beam quality and reduced optical damage to the emitting surface, the gain medium of the proposed $\mu$ laser was designed to be composed of multistripe index-guided laser arrays vertically sandwiched between $\mathrm{SiO}_{2}$ (substrate) and air. The structure thus operates as a dielectric waveguide that ensures efficient confinement of photons, overlapping with the active optical gain region. However, the number and type of oscillating resonator modes in the $\mu$-laser depend on the length of gain medium between mirrors, the amount of material gain and losses (scattering and reabsorption). In this regard, two $\mu$-laser structures with different lengths of gain media (40 and $20 \mu \mathrm{m}$ long) were designed and lasing capabilities were evaluated.

To investigate the lasing performance of the patterned structures, we conducted PL measurements where the patterned ( $40 \mu \mathrm{m}$ in length) substrates were excited with a pulsed laser beam of $400 \mathrm{~nm}$ wavelength, $\sim 110 \mathrm{fs}$ pulse width, and $1 \mathrm{kHz}$ pulse rate. The PL emission at different pump intensities was collected from the side of the substrate with an optical fiber. The resulting spectra of the deep-patterned CQWs are displayed in Figure 3a. For low pump intensities, a broad spectrum of spontaneous emission is observed, whereas narrow spectral features with fwhm around $0.6 \mathrm{~nm}$ appear above $21.0 \mu \mathrm{J} / \mathrm{cm}^{2}$. These features are attributed to the lasing modes because of their ultrasmall FHWM accompanied by the superlinear increase in the intensity at the spectral peaks of these features beyond the threshold as shown in Figure $3 \mathrm{~b}$. After the appearance of the first lasing mode at $\sim 662 \mathrm{~nm}$, the second one emerges at $\sim 650 \mathrm{~nm}$ with a threshold of $23.0 \mu \mathrm{J} /$ $\mathrm{cm}^{2}$. By increasing the pump intensity even further beyond 70 $\mu \mathrm{J} / \mathrm{cm}^{2}$, a third peak at $\sim 638 \mathrm{~nm}$ is observed. The spectral spacing of these modes are about $12 \mathrm{~nm}$, which agrees with the expected mode spacing of the designed CQW patterns. The simulated cavity mode intensity profile for the dominant mode with $=664 \mathrm{~nm}$ is shown in Figure 3c.

A possible approach to attain single-mode lasing is to reduce the length of cavity. However, this will inevitably increase the lasing threshold of the $\mu$-laser and require significantly high pumping intensity. Single-frequency operation is desirable but at the same time more difficult to achieve as this requires a cavity with precisely tuned free spectral range that permits only one mode across the gain spectrum of the emitters. Thereby, the same experiment was conducted using the shorter gain medium laser ( $20 \mu \mathrm{m}$ in length). Figure $3 \mathrm{~d}$ shows the pump intensity dependent spectra of the deep-patterned CQWs. We observe the lasing behavior at a threshold of $147 \mu \mathrm{J} / \mathrm{cm}^{2}$, which is evident from the spectral narrowing of the PL spectrum beyond the threshold as well as the superlinear increase of the intensity at the wavelength of interest as seen in
Figure 3e. Simulated cavity mode intensity profiles for the dominant mode at $\sim 663 \mathrm{~nm}$ is shown in Figure $3 \mathrm{f}$.

Finally, to assess the reliability and feasibility of the proposed direct deep-patterning method in optoelectronic applications, our procedure is applied to different group of colloidal particles including colloidal quantum dots (QDs) and metal-oxide nanoparticles and by adapting (CMOS)-compatible processes by changing electron beam lithography to photolithography. The mentioned nanoparticles are commonly used in pixelated lighting for liquid crystal displays. The highresolution dual-color and multicolor lithographic patterning is also shown with our direct deep-patterning fabrication method. Figure $4 \mathrm{a}$ shows bicolor patterning of red-emitting CdSe/ $\mathrm{CdZnS}$ CQWs and green-emitting CdSe/CdS QDs with electron beam lithography, which may provide a new and unique approach also for realizing efficient micro-LED displays. Ultrafine submicron-scale pixelated, high-efficiency, multicolor light sources integrated on a single chip are required by the display technologies of tomorrow. Figure $4 \mathrm{~b}$ shows the bicolor patterning of two different NCs with photolithography (see the Supporting for detail). Figure 4c, d show high-quality, ultrathick patterns of green-emitting $\mathrm{QDs}$ and red-emitting CQWs that patterned by EBL with high-aspect ratios, which are the highest values in their class reported to date, to the best of our knowledge. Fabrication of thick NC-based Bragg gratings with small periods and high refractive index have so far not been reported. Figure 4e shows deep-patterned CQW bull's-eye geometry with a pitch of $650 \mathrm{~nm}$. These bull's-eye geometries favor enhanced light extraction from light-emitting diodes and for demonstrating annular Bragg lasers. Figure $4 \mathrm{f}$ displays deep-patterned Au NPs. Detailed results are shown in Figure S4.

\section{CONCLUSION}

In conclusion, we have successfully developed a direct deepnanopatterning technique based on EBL used in conjunction with UV-induced ligand exchange. This method allows to pattern semiconductor NCs and metal nanoparticles. Using this approach, we fabricated the first high-quality fully colloidal self-resonant $\mu$-laser, which operates with a low optical pump threshold at room temperature, with in-plane emission. The fabricated $\mu$-laser is an excellent candidate to be used in integrated photonic circuits for practical applications such as lab-on-a-chip and optofluidics. The design and technique for fabricating all-colloidal self-resonating $\mu$-lasers presented in this work can be a future direction for ongoing works on colloidalbased microlasers operating under ns-CW-pumping.

\section{ASSOCIATED CONTENT}

\section{Supporting Information}

The Supporting Information is available free of charge at https://pubs.acs.org/doi/10.1021/acs.nanolett.1c00464.

Chemicals and materials, surface morphology and height characterization of deep-patterned structures with atomic force microscopy (AFM), photoluminescence quantum efficiency (PLQE) of the CQW films before and after the fabrication process, microscale patterning of green-emitting $\mathrm{CdSe} / \mathrm{ZnS}$ QDs, red-emitting $\mathrm{CdSe} /$ $\mathrm{CdZnS}$ CQWs and metal NPs, process maturity assessment of the proposed patterning method (PDF) 


\section{AUTHOR INFORMATION}

\section{Corresponding Author}

Hilmi Volkan Demir - Department of Electrical and Electronics Engineering Department of Physics, UNAM-Institute of Materials Science and Nanotechnology, Bilkent University, Ankara 06800, Turkey; LUMINOUS! Centre of Excellence for Semiconductor Lighting and Displays, Centre of Optical Fiber Technology, The Photonics Institute, School of Electrical and Electronic Engineering, School of Physical and Mathematical Sciences, Nanyang Technological University, Singapore 639798, Singapore; Email: hvdemir@ntu.edu.sg, volkan@bilkent.edu.tr

\section{Authors}

Negar Gheshlaghi - Department of Electrical and Electronics Engineering Department of Physics, UNAM-Institute of Materials Science and Nanotechnology, Bilkent University, Ankara 06800, Turkey; 이이.org/0000-0003-1036-3377

Sina Foroutan-Barenji - Department of Electrical and Electronics Engineering Department of Physics, UNAM-Institute of Materials Science and Nanotechnology, Bilkent University, Ankara 06800, Turkey; 이이.org/ 0000-0003-0623-8987

Onur Erdem - Department of Electrical and Electronics Engineering Department of Physics, UNAM-Institute of Materials Science and Nanotechnology, Bilkent University, Ankara 06800, Turkey

Yemliha Altintas - Department of Electrical and Electronics Engineering Department of Physics, UNAM-Institute of Materials Science and Nanotechnology, Bilkent University, Ankara 06800, Turkey; Department of Materials Science and Nanotechnology, Abdullah Gul University, Kayseri 38080, Turkey

Farzan Shabani - Department of Electrical and Electronics Engineering Department of Physics, UNAM-Institute of Materials Science and Nanotechnology, Bilkent University, Ankara 06800, Turkey

Muhammad Hamza Humayun - Department of Electrical and Electronics Engineering Department of Physics, UNAM-Institute of Materials Science and Nanotechnology, Bilkent University, Ankara 06800, Turkey

Complete contact information is available at: https://pubs.acs.org/10.1021/acs.nanolett.1c00464

\section{Author Contributions}

H.V.D. conceived the main idea of this study and supervised the research at all stages. N.G. developed the fabrication steps and fabricated the designed structures. N.G. \& S.F.-B. designed and implemented the self-resonant $\mu$-lasers and O.E. and N.G. conducted the laser measurements. Y.A. and F.S. synthesized the engineered CQWs and characterized the optical properties of the samples in the solution form. N.G. and M.H.H. performed the ellipsometry and TRF measurements. N.G. and H.V.D. wrote the manuscript with inputs from all coauthors. All authors have given approval to the final version of the manuscript.

Notes

The authors declare no competing financial interest.

\section{ACKNOWLEDGMENTS}

This research was supported in part by the National Research Foundation, Prime Minister's Office, Singapore, under its
Investigatorship Program (NRF-NRFI2016-08) and the Singapore Agency for Science, Technology and Research (A*STAR) SERC Pharos Program under Grant 1527300025. The authors also acknowledge financial support from TÜBITAK through 115E679, 115F297, and 117E713 programs. The authors thank Mr. Mustafa Guler and Mr. Ovunc Karakurt for their assistance in TEM imaging, Dr. Gokce Celik for her help on the ellipsometric measurements, Mr. Semih Bozkurt for his support on the AFM characterization, and Mr. Bilge Yagci for his assistance in optical characterization. O.E. acknowledges TÜBITAK for financial support through the BIDEB-2211 program. H.V.D. gratefully acknowledges TÜBA.

\section{REFERENCES}

(1) Fang, Y.; Sun, M. Nanoplasmonic waveguides: towards applications in integrated nanophotonic circuits. Light: Sci. Appl. 2015, 4, e294.

(2) Gramotnev, D. K.; Bozhevolnyi, S. I. Plasmonics beyond the diffraction limit. Nat. Photonics 2010, 4, 83-91.

(3) Chen, R.; Tran, T. D.; Ng, K. W.; Ko, W. S.; Chuang, L. C.; Sedgwick, F. G.; Chang-Hasnain, C. J. N. P. Nanolasers grown on silicon. Nat. Photonics 2011, 5, 170-175.

(4) Alivisatos, A. P. Semiconductor clusters, nanocrystals, and quantum dots. Science 1996, 271, 933-937.

(5) Dabbousi, B. O.; Rodriguez-Viejo, J.; Mikulec, F. V.; Heine, J. R.; Mattoussi, H.; Ober, R.; Jensen, K. F.; Bawendi, M. G. (CdSe) ZnS core- shell quantum dots: synthesis and characterization of a size series of highly luminescent nanocrystallites. J. Phys. Chem. B 1997, $101,9463-9475$.

(6) Milliron, D. J.; Hughes, S. M.; Cui, Y.; Manna, L.; Li, J.; Wang, L.-W.; Alivisatos, A. P. Colloidal nanocrystal heterostructures with linear and branched topology. Nature 2004, 430, 190-195.

(7) Ithurria, S.; Tessier, M. D.; Mahler, B.; Lobo, R. P. S. M.; Dubertret, B.; Efros, Al. L. Colloidal nanoplatelets with twodimensional electronic structure. Nat. Mater. 2011, 10, 936-941.

(8) Dang, C.; Lee, J.; Breen, C.; Steckel, J. S.; Coe-Sullivan, S.; Nurmikko, A. Red, green and blue lasing enabled by single-exciton gain in colloidal quantum dot films. Nat. Nanotechnol. 2012, 7, 335339.

(9) Guzelturk, B.; Kelestemur, Y.; Gungor, K.; Yeltik, A.; Akgul, M. Z.; Wang, Y.; Chen, R.; Dang, C.; Sun, H.; Demir, H. V. Stable and low-threshold optical gain in $\mathrm{CdSe} / \mathrm{CdS}$ quantum dots: an allcolloidal frequency up-converted laser. Adv. Mater. 2015, 27, 27412746.

(10) Grim, J. Q.; Christodoulou, S.; Di Stasio, F.; Krahne, R.; Cingolani, R.; Manna, L.; Moreels, I. Continuous-wave biexciton lasing at room temperature using solution-processed quantum wells. Nat. Nanotechnol. 2014, 9, 891-895.

(11) Maskoun, J.; Gheshlaghi, N.; Isik, F.; Delikanli, S.; Erdem, O.; Erdem, E. Y.; Demir, H. V. Optical Microfluidic Waveguides and Solution Lasers of Colloidal Semiconductor Quantum Wells. Adv. Mater. 2021, 33, 2170070-2170076.

(12) Wang, Y.; Leck, K. S.; Ta, V. D.; Chen, R.; Nalla, V.; Gao, Y.; He, T.; Demir, H. V.; Sun, H. Blue liquid lasers from solution of $\mathrm{CdZnS} / \mathrm{ZnS}$ ternary alloy quantum dots with quasi-continuous pumping. Adv. Mater. 2015, 27, 169-175.

(13) Kazes, M.; Lewis, D. Y.; Ebenstein, Y.; Mokari, T.; Banin, U. Lasing from semiconductor quantum rods in a cylindrical microcavity. Adv. Mater. 2002, 14, 317-321.

(14) Prins, F.; Kim, D. K.; Cui, J.; De Leo, E.; Spiegel, L. L.; McPeak, K. M.; Norris, D. J. Direct patterning of colloidal quantumdot thin films for enhanced and spectrally selective out-coupling of emission. Nano Lett. 2017, 17, 1319-1325.

(15) Gao, Y.; Tobing, L. Y.; Kiffer, A. L.; Zhang, D. H.; Dang, C.; Demir, H. V. Azimuthally polarized, circular colloidal quantum dot 
laser beam enabled by a concentric grating. ACS Photonics 2016, 3, 2255-2261.

(16) Kozlov, O. V.; Park, Y.-S.; Roh, J.; Fedin, I.; Nakotte, T.; Klimov, V. I. Sub-single-exciton lasing using charged quantum dots coupled to a distributed feedback cavity. Science 2019, 365, 672-675.

(17) Zhu, Y.; Xie, W.; Bisschop, S.; Aubert, T.; Brainis, E.; Geiregat, P.; Hens, Z.; Van Thourhout, D. On-chip single-mode distributed feedback colloidal quantum dot laser under nanosecond pumping. ACS Photonics 2017, 4, 2446-2452.

(18) Yang, Z.; Pelton, M.; Fedin, I.; Talapin, D. V.; Waks, E. A room temperature continuous-wave nanolaser using colloidal quantum wells. Nat. Commun. 2017, 8, 143-151.

(19) Wang, Y.; Fedin, I.; Zhang, H.; Talapin, D. V. Direct optical lithography of functional inorganic nanomaterials. Science 2017, 357, 385-388.

(20) Wang, Y.; Pan, J. A.; Wu, H.; Talapin, D. V. Direct wavelengthselective optical and electron-beam lithography of functional inorganic nanomaterials. ACS Nano 2019, 13, 13917-13931.

(21) Miszta, K.; Greullet, F.; Marras, S.; Prato, M.; Toma, A.; Arciniegas, M.; Manna, L.; Krahne, R. Nanocrystal film patterning by inhibiting cation exchange via electron-beam or X-ray lithography. Nano Lett. 2014, 14, 2116-2122.

(22) Kim, W. J.; Kim, S. J.; Lee, K.-S.; Samoc, M.; Cartwright, A. N.; Prasad, P. N. Robust microstructures using UV photopatternable semiconductor nanocrystals. Nano Lett. 2008, 8, 3262-3265.

(23) Kim, L.; Anikeeva, P. O.; Coe-Sullivan, S. A.; Steckel, J. S.; Bawendi, M. G.; Bulovic, V. Contact printing of quantum dot lightemitting devices. Nano Lett. 2008, 8, 4513-4517.

(24) Kim, T.-H.; Cho, K.-S.; Lee, E. K.; Lee, S. J.; Chae, J.; Kim, J. W.; Kim, D. H.; Kwon, J.-Y.; Amaratunga, G.; Lee, S. Y.; et al. Fullcolour quantum dot displays fabricated by transfer printing. Nat. Photonics 2011, 5, 176-182.

(25) Kiremitler, N. B.; Torun, I.; Altintas, Y.; Patarroyo, J.; Demir, H. V.; Puntes, V. F.; Mutlugun, E.; Onses, M. S. Writing chemical patterns using electrospun fibers as nanoscale inkpots for directed assembly of colloidal nanocrystals. Nanoscale 2020, 12, 895-903.

(26) Erdem, O.; Foroutan, S.; Gheshlaghi, N.; Guzelturk, B.; Altintas, Y.; Demir, H. V. Thickness-Tunable Self-Assembled Colloidal Nanoplatelet Films Enable Ultrathin Optical Gain Media. Nano Lett. 2020, 20, 6459-6465.

(27) Elshaari, A. W.; Zadeh, I. E.; Fognini, A.; Reimer, M. E.; Dalacu, D.; Poole, P. J.; Zwiller, V.; Jöns, K. D. On-chip single photon filtering and multiplexing in hybrid quantum photonic circuits. Nat. Commun. 2017, 8, 1-8.

(28) Foroutan-Barenji, S.; Erdem, O.; Delikanli, S.; Yagci, H. B.; Gheshlaghi, N.; Altintas, Y.; Demir, H. V. Single-mode lasing from a single $7 \mathrm{~nm}$ thick monolayer of colloidal quantum wells in a monolithic microcavity. Laser Photonics Rev. 2021, 15, 20004792000486.

(29) Zavelani-Rossi, M.; Krahne, R.; Della Valle, G.; Longhi, S.; Franchini, I. R.; Girardo, S.; Scotognella, F.; Pisignano, D.; Manna, L.; Lanzani, G.; Tassone, F. Self-assembled CdSe/CdS nanorod microlasers fabricated from solution by capillary jet deposition. Laser Photonics Rev. 2012, 6, 678-683.

(30) Schafer, J.; Mondia, J. P.; Sharma, R.; Lu, Z.; Susha, A.; Rogach, A. L.; Wang, L. J. Quantum dot microdrop laser. Nano Lett. 2008, 8, 1709-1712.

(31) Wang, Y.; Ta, V. D.; Leck, K. S.; Tan, B. H. I.; Wang, Z.; He, T.; Ohl, C. D.; Demir, H. V.; Sun, H. Robust whispering-gallery-mode microbubble lasers from colloidal quantum dots. Nano Lett. 2017, 17, 2640-2646.

(32) Rossinelli, A. A.; Rojo, H.; Mule, A. S.; Aellen, M.; Cocina, A.; De Leo, E.; Schäublin, R.; Norris, D. J. Compositional Grading for Efficient and Narrowband Emission in CdSe-Based Core/Shell Nanoplatelets. Chem. Mater. 2019, 31, 9567-9578.

(33) Le Feber, B.; Prins, F.; De Leo, E.; Rabouw, F. T.; Norris, D. J. Colloidal-quantum-dot ring lasers with active color control. Nano Lett. 2018, 18, 1028-1034.
(34) Xie, W.; Stoferle, T.; Raino, G.; Aubert, T.; Bisschop, S.; Zhu, Y.; Mahrt, R. F.; Geiregat, P.; Brainis, E.; Hens, Z.; Van Thourhout, D. On-Chip Integrated Quantum-Dot-Silicon-Nitride Microdisk Lasers. Adv. Mater. 2017, 29, 1604866.

(35) Adachi, M. M.; Fan, F.; Sellan, D. P.; Hoogland, S.; Voznyy, O.; Houtepen, A. J.; Parrish, K. D.; Kanjanaboos, P.; Malen, J. A.; Sargent, E. H. Microsecond-sustained lasing from colloidal quantum dot solids. Nat. Commun. 2015, 6, 1-8.

(36) Chen, C.; Yuan, J.; Wan, L.; Chandrahalim, H.; Chen, Z.; Nishimura, N.; Takeda, H.; Yoshioka, H.; Liu, W.; Oki, Y. J. O. L.; et al. Demonstration of on-chip quantum dot microcavity lasers in a molecularly engineered annular groove. Opt. Lett. 2019, 44, 495-498.

(37) Altintas, Y.; Quliyeva, U.; Gungor, K.; Erdem, O.; Kelestemur, Y.; Mutlugun, E.; Kovalenko, M. V.; Demir, H. V. Highly Stable, Near-Unity Efficiency Atomically Flat Semiconductor Nanocrystals of $\mathrm{CdSe} / \mathrm{ZnS}$ Hetero-Nanoplatelets Enabled by ZnS-Shell Hot-Injection Growth. Small 2019, 15, 1804854.

(38) Altintas, Y.; Gungor, K.; Gao, Y.; Sak, M.; Quliyeva, U.; Bappi, G.; Mutlugun, E.; Sargent, E. H.; Demir, H. V. Giant alloyed hot injection shells enable ultralow optical gain threshold in colloidal quantum wells. ACS Nano 2019, 13, $10662-10670$.

(39) Pallavicini, P.; Dacarro, G.; Galli, M.; Patrini, M. Spectroscopic evaluation of surface functionalization efficiency in the preparation of mercaptopropyltrimethoxysilane self-assembled monolayers on glass. J. Colloid Interface Sci. 2009, 332, 432-438.

(40) Mentzel, T. S.; Wanger, D. D.; Ray, N.; Walker, B. J.; Strasfeld, D.; Bawendi, M. G.; Kastner, M. A. Nanopatterned electrically conductive films of semiconductor nanocrystals. Nano Lett. 2012, 12, 4404-4408.

(41) Fafarman, A. T.; Hong, S.-H.; Caglayan, H.; Ye, X.; Diroll, B. T.; Paik, T.; Engheta, N.; Murray, C. B.; Kagan, C. R. Chemically tailored dielectric-to-metal transition for the design of metamaterials from nanoimprinted colloidal nanocrystals. Nano Lett. 2013, 13, 350357.

(42) Jun, S.; Jang, E.; Park, J.; Kim, J. Photopatterned semiconductor nanocrystals and their electroluminescence from hybrid light-emitting devices. Langmuir 2006, 22, 2407-2410.

(43) Webber, D. H.; Brutchey, R. L. Nanocrystal ligand exchange with 1,2,3, 4-thiatriazole-5-thiolate and its facile in situ conversion to thiocyanate. Dalton Trans. 2012, 41, 7835-7838.

(44) Fafarman, A. T.; Koh, W. K.; Diroll, B. T.; Kim, D. K.; Ko, D. K.; Oh, S. J.; Ye, X.; Doan-Nguyen, V.; Crump, M. R.; Reifsnyder, D. C.; Murray, C. B.; Kagan, C. R. Thiocyanate-capped nanocrystal colloids: vibrational reporter of surface chemistry and solution-based route to enhanced coupling in nanocrystal solids. J. Am. Chem. Soc. 2011, 133, 15753-15761.

(45) Liu, M.; Voznyy, O.; Sabatini, R.; Garcia de Arquer, F. P.; Munir, R.; Balawi, A. H.; Lan, X.; Fan, F.; Walters, G.; Kirmani, A. R.; Hoogland, S.; Laquai, F.; Amassian, A.; Sargent, E. H. Hybrid organicinorganic inks flatten the energy landscape in colloidal quantum dot solids. Nat. Mater. 2017, 16, 258-263.

(46) Lhuillier, E.; Keuleyan, S.; Zolotavin, P.; Guyot-Sionnest, P. J. Mid-Infrared $\mathrm{HgTe} / \mathrm{As} 2 \mathrm{~S} 3$ Field Effect Transistors and Photodetectors. Adv. Mater. 2013, 25, 137-141.

(47) Choi, J.-H.; Wang, H.; Oh, S. J.; Paik, T.; Sung, P.; Sung, J.; Ye, X.; Zhao, T.; Diroll, B. T.; Murray, C. B.; Kagan, C. R. Exploiting the colloidal nanocrystal library to construct electronic devices. Science 2016, 352, 205-208.

(48) Diroll, B. T.; Gaulding, E. A.; Kagan, C. R.; Murray, C. B. Spectrally resolved dielectric functions of solution-cast quantum dot thin films. Chem. Mater. 2015, 27, 6463-6469.

(49) Dement, D. B.; Puri, M.; Ferry, V. E. Determining the complex refractive index of neat $\mathrm{CdSe} / \mathrm{CdS}$ quantum dot films. J. Phys. Chem. C 2018, 122, 21557-21568. 